\title{
Discutir el campo del capital social desde un enfoque transdisciplinario
}

\author{
Gardy Augusto Bolívar Espinoza
}

Universidad Autónoma Metropolitana, Unidad Azcapotzalco, Ciudad de México, México. Email: abe88@hotmail.com

\section{Leonel Flores Vega}

Universidad Autónoma Metropolitana, Unidad Xochimilco, Ciudad de México, México.

Email: hemaus@live.com.mx.

Resumen: Este artículo expone algunos antecedentes sobre el origen y las vertientes actuales del campo del capital social en las ciencias sociales en tanto instrumento analítico de la realidad social. Trata de los fundadores y de sus posiciones, así como de los trabajos emprendidos desde las organizaciones internacionales, especialmente, el Banco Mundial. Discute sobre las dimensiones e indicadores, señalando los principales aportes y los desafíos necesarios de considerar.

Palabras clave: ciencias sociales, capital social, relaciones sociales.

\section{Discussing the field of social capital from a transdisciplinary approach}

Abstract: This article states some backgrounds on the origin and current aspects on the field of social capital on social sciences as analytical tool of social reality. It deals with the founders and their positions, as well as of the works undertaken by international organizations, especially the World Bank. It discusses about the dimensions and indicators, highlighting the main contributions and the challenges that must be considered.

Key words: social sciences, social capital, social relations.

\section{Discutir o domínio do capital social a partir de uma abordagem transdisciplinar}

Resumo: Este artigo presenta algumas informações sobre a origem, os aspectos atuais da área de capital social nas ciências sociais como ferramenta analítica da realidade social. Trata sobre os fundadores e as suas posições e os trabalhos realizados desde organizações internacionais, especialmente o Banco Mundial. Discute sobre as dimensões e indicadores, destacando as principais contribuições e os desafios que devem ser considerados.

Palavras-chave: ciências sociais, capital social, relações sociais. 


\section{Introducción}

A finales del siglo pasado se consolidó el campo de estudio denominado "Capital Social”, compuesto de disciplinas como la economía, la sociología, la ciencia política, la antropología, entre otros. Surge como un instrumento analítico para explicar el papel que desempeñan las relaciones sociales en los procesos de desarrollo de las sociedades contemporáneas. Crecientes estudios empíricos se han desarrollado en distintas temáticas, buscando encontrar elementos que permitan transformas la confianza, la solidaridad, reciprocidad en bienes tangibles y en políticas públicas que otorguen mejores condiciones de vida.

La misión del trabajo es mostrar cómo el capital social se formó como un campo de estudio, iniciado con algunos antecedentes en las ciencias sociales, seguido de los fundadores formales y organismos internacionales. Sostendremos que a pesar de la falta de acuerdo y claridad del concepto, y de los estudios de caso que pareciera pueden decir cualquier cosa de cualquier tema, han contribuido positivamente.

El capital social incluye "el ambiente social y político que conforma la estructura social": relaciones y estructuras institucionales formalizadas que permiten el desarrollo de normas a través del régimen político y el gobierno, el sistema judicial y la aplicación del el derecho, y las libertades civiles y políticas.

Este punto de vista no sólo toma en cuenta las virtudes y los vicios del capital social, y la importancia de la formación de relaciones dentro y entre comunidades, sino también reconoce que la capacidad de varios grupos sociales para actuar en su propio interés depende crucialmente del apoyo (o la falta de apoyo) que reciban del estado y del sector privado. De la misma manera, el estado depende de la estabilidad social y del apoyo popular. En resumen, el desarrollo económico y social prospera cuando los representantes del estado, del sector empresarial y de la sociedad civil crean foros a través de los cuales puedan identificar y alcanzar metas comunes.

\section{La construcción del campo}

\section{Dos vías lo "Social” y la del “capital”}

Varios estudios concuerdan que el concepto de Capital social ha sido utilizado por distintos autores, en distintas épocas, pero que Lyda Judson Hanifan lo utiliza por primera vez en 1916 cuando describe algunos centros comunitarios en escuelas rurales. Posteriormente, en la década de los sesenta, Jane Jacobs lo vuelve a usar para explicar las densas redes de las áreas urbanas de uso mixto que constituían una forma de capital social que desafiaba la seguridad pública. En la década de los setenta Glenn Loury e Iván Light emplean la expresión para analizar el problema del desarrollo 
económico de las áreas centrales de las grandes urbes.

Los usos del concepto Capital social tienen por lo menos dos dimensiones. La primera, relacionada con el uso de los contenidos del concepto actual pero que viene de la tradición teórica y que no se denominaba explícitamente como capital social. La segunda, tal cual como ahora es concebida siguiendo los pasos de Bourdieu, Coleman y Putnam, que se consolidan como los principales teóricos del campo que se inaugura en la década de los ochenta del siglo pasado.

Desde la tradición en las ciencias sociales muchos son los que pueden ubicarse en este campo, desde la filosofía clásica hasta los más variados iniciadores de la sociología. Fundamentalmente habría que mencionar al concepto de “capital” y el de "social” tal cual se entiende en El Capital de Karl Marx. También Alexis de Tocqueville es a menudo citado al destacar su referencia al "arte de la asociación”( Fukuyama, 1999).

Por otra parte, desde la corriente americana, el estructural funcionalismo de Parsons sirve de terreno criticado donde surge por negación el concepto. Se le critica al funcionalismo estructural, por el excesivo énfasis en las normas en detrimento de los motivos de los actores, y, por su énfasis en las determinación estructurales de “arriba hacia abajo” que descuidan el papel de las estructuras y relaciones sociales de nivel individual. Homans, Gouldner persisten en esta discusión y los sociólogos vinculados a la etnometodología y el interaccionismo simbólico, como Goffman, Blumer, Cooley y Rosenberg, entre otros, desarrollan parte importante del bagaje de pensadores que destacan la relevancia de las relaciones sociales, en particular, de solidaridad y reciprocidad, así como de los valores, los sentimientos y las normas que suelen asociarse con ellas (Cuéllar Saavedra \& Bolívar Espinoza, 2009).

Una vía para contribuir a la comprensión del significado del capital social es descomponer el término en sus elementos principales: "capital” y “social”. Sin embargo, la conjunción de estos dos términos no significa que está libre de riesgos de contradicción. Respecto del primero, arranca de la tradición de la economía clásica y específicamente de la noción de riqueza y, posteriormente, de la teoría del valor. Como nos recuerda Fukuyama (1999) la motivación que tienen los individuos cuando se reúnen en el mercado, desde el punto de vista de la economía, es para intercambiar bienes y servicios. Este punto de vista refleja la corriente neoclásica de la economía con el modelo racional del comportamiento humano, supone que los individuos actúan de forma egoísta al actuar buscando maximizar su utilidad al relacionarse con otros individuos. En tanto, desde el punto de vista de la sociología -que es al parecer el de mayor fuerza- se impulsa la idea de lo "social”, que surge de la sociología clásica se traduce en la noción de solidaridad cuyo pasado se remonta a la filosofía clásica ${ }^{2}$, en donde se utiliza como medio por excelencia de las relaciones sociales y la acción colectiva.

Del amplio el abanico de autores relacionados sustantivamente con 
el capital social, dos autores son inevitables en la teoría social para referirse a las relaciones sociales: Durkheim y Weber. Durkheim en la División social del trabajo, plantea dos cuestiones: la primera, que a menor división del trabajo la vinculación entre los individuos (solidaridad mecánica) se incrementa; y, la segunda que la modernización erosiona tales vínculos y crea obligaciones que obligan al individuo a una interdependencia funcional (solidaridad orgánica). La cohesión es un momento de la solidaridad social necesaria para que los integrantes de una sociedad sigan ligados a ella con una fuerza análoga a la generada por la solidaridad mecánica, lo cual requiere que sus lazos se hagan más fuertes e incluyan ideas y sentimientos que los unan (Durkheim, 2005).

Desde la perspectiva weberiana, la acción social es definida como: una conducta humana (consistente en un hacer externo o interno o bien, en un omitir o permitir) siempre que el sujeto o los sujetos de la acción enlacen a ella un sentido subjetivo. La “acción social” por tanto, es una acción en donde el sentido mentado por el sujeto o sujetos está referido a la conducta de otros, orientándose por ésta en su desarrollo (Weber, 2000). La significación de la acción social es que siempre está dirigida a otras personas y no es resultado de un estímulo cualquiera.

\section{Cuadro 1}

\section{Principales Teóricos del Capital social por año.}

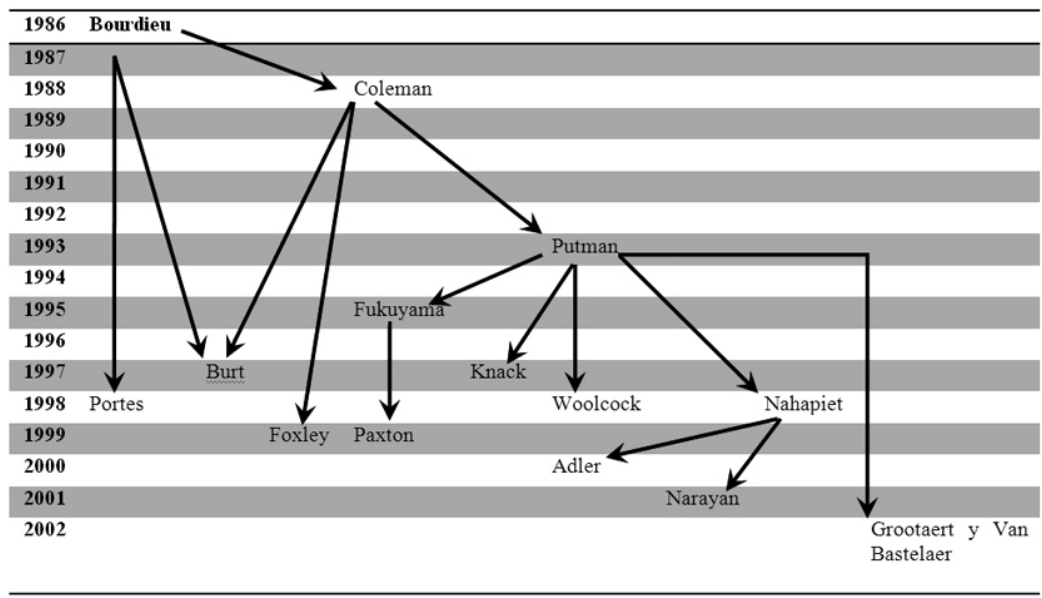

Fuente: (Claridge, 2004) y (Gallo Rivera \& Garrido Yserte, 2009, pág. 11)

La inauguración del nuevo campo del capital social abre nuevas posturas y reflexiones respecto de las relaciones sociales que sintetizan posturas surgidas desde la economía, la sociología, la ciencia política y la antropología. Los referentes presentados en el Cuadro 1, muestra a los 
principales autores, que han desarrollado alguna parte del campo ${ }^{3}$. Reseñar la parte sustantiva de sus planteamientos no puede realizarse en este texto pero en el siguiente apartado se hace un breve referencia a sus tres principales fundadores, Bourdieu, Coleman, Putnam.

\section{Dos corrientes (estructurales/Culturales)}

Aunque destacan dos corriente principales: las "definiciones estructurales” fundamentalmente referidas a Bourdieu y Coleman y las definiciones culturales cuyo mejor representante sería Putnam ${ }^{4}$. La primera se ubica, según Bourdieu, en "el agregado de recursos reales o potenciales que se vinculan con la posesión de una red duradera de relaciones más o menos institucionalizadas de conocimiento y reconocimiento mutuo": acceso a la información, obligaciones que se desprenden de la confianza mutua, o aprovechamiento de las normas sociales cooperativas. La referencia material de este enfoque se encuentra en las redes. Estas tiene historia y continuidad puede estar cerradas, unidireccionales, simétricas, horizontales o, sus contrarios; los vinculos pueden ser fuertes o débiles o frecuentes, con puentes hacia otras redes, o volcados a su interior, etc.

La segunda, refiere a un fenómeno subjetivo: valores y actitudes cuyo centro es la confianza generalizada, vinculada a un "juicio moral" conectado con la "cultura política”. Se podría decir que para Putnam la virtud cívica es capital social. Los temas que recurren a este concepto son variados y amplios: la creación de Capital Social; la efectividad de las instituciones democráticas; el desarrollo económico; y la acción colectiva. En las investigaciones sobre capital social este aparece ya sea como variable dependiente o independiente: ya sea el conjunto de causas y procesos implicados en su formación; o sus consecuencias.

Pierre Bourdieu considera que el capital social es:

“es el agregado de los actuales o potenciales recursos que están relacionados con la posesión de una red perdurable de relaciones más o menos institucionalizadas de conocimiento y reconocimiento mutuo -en otras palabras, con la pertenencia a un grupo-que le brinda a cada uno de los miembros el respaldo del capital socialmente adquirido, una credencial que les permite acreditarse, en los diversos sentidos de la palabra” (Bourdieu, 1986, pág. 249).

El capital social se conformaría por redes sociales, que los individuos establecen a fin de obtener beneficios, para ello, es necesario que la red sea estable, útil y movible para cuando tengamos que recurrir a ella. El grado de capital social que se posea dependerá de la capacidad que tengan los individuos de expandir sus redes y del volumen de capital social ostentado por las personas con las que se relaciona. Esto dotará a los individuos de mayores y mejores oportunidades en la reproducción del capital económico y cultural (Bourdieu, 1986). 
James Coleman (1988) con su artículo "Social Capital in the Creation of Human Capital" resalta el papel de la confianza, de la reciprocidad en las relaciones sociales como medios para obtener mayor el capital social. Estas relaciones deben estar mediadas por diversas normas que favorecen determinadas conductas y constriñen a otras, éstas deben ser respetadas si se pretende obtener ayuda cuando se tiene un problema relacionado con los bienes públicos en una comunidad, de ahí que el capital social también pueda beneficiar a colectividades y esté, por ello, dotado de una naturaleza colectiva.

Coleman desarrolla su perspectiva desde el punto de vista funcional, pone énfasis, no en lo que es, sino en los beneficios que puede brindar a los individuos. Es decir, el valor que tiene para los individuos los distintos recursos a los que puede recurrir para facilitarse el acceso a sus intereses. Esto consolida la estructura social -fundamento de la sociología-donde los individuos no están aislados, sino que están relacionados con independencia de sus acciones, además de ser coercitivos, pero al mismo tiempo facilita las condiciones para que pueda actuar y beneficiarse de los atributos que posee dicha estructura (Coleman, 1990).

Coleman identifica varias formas de capital social: las obligaciones y las expectativas, que se refieren al intercambio de favores, más o menos formal; el empleo de amigos y conocidos como fuentes de información; las normas, sean internas al individuo o fundadas en un sistema de incentivos y sanciones; las relaciones de autoridad y las organizaciones. Coleman señala que el capital social es un bien público por cuanto sus beneficios no sólo son captados por los actores involucrados en una relación social, sino por otros; por ejemplo, un vecino puede ser apático frente a la organización comunitaria de su barrio y aun así, disfrutar de los beneficios de las acciones de esa organización. (Coleman, 1990, págs. 315-318).

En lo que se refiere a Robert Putnam, su mayor aporte surge con su famoso estudio en Italia, en el que muestra algunas evidencias empíricas sobre la relación que existe entre los componentes del capital social -la confianza, las normas de reciprocidad y las redes- y un alto desempeño gubernamental (Putnam, 1993). En el debate sobre la relación entre asociatividad, confianza y compromiso cívico, Robert Putnam ha sostenido que las asociaciones cívicas o de voluntariado constituyen espacios de interacción social en los que se facilita el aprendizaje de actitudes y conductas cooperadoras, incrementan la interacción cara a cara y crean condiciones para el desarrollo de la confianza, y que la confianza generada dentro de ellas, así como las experiencias de cooperación de sus miembros, tienden a generalizarse al conjunto de la sociedad (Putman, 2003).

Reconociendo el papel fundamental que tienen las normas en la sociedad, los individuos generan un ambiente de confianza, con normas de reciprocidad claras, redes, asociatividad, confianza y compromiso cívico que mejoran la eficacia de la sociedad facilitando la acción coordinada. En este sentido, las organizaciones que no dependen del gobierno coadyuvan sustancialmente a la formación de capital social precisamente porque gene- 
ran distintos tipos de interacciones, facilitando procesos de cooperación solidarios que generan confianza, además hacen accesibles los bienes y servicios públicos que el gobierno no logra brindar (Putnam, 1993).

En su investigación que compara el Norte y el Sur de Italia, Putnam (1993) examina el capital social en términos del grado de participación de la sociedad civil como medida del número de votantes, lectores de periódicos, miembros de grupos corales y clubes de fútbol, y confianza en las instituciones públicas. El norte de Italia, donde todos estos indicadores son altos, muestra tasas significativamente más elevadas de gobernabilidad, de rendimiento institucional y de desarrollo, aun cuando se controlan otros factores ortodoxos. En su trabajo sobre los Estados Unidos, Putnam (1995, 1998) utiliza un enfoque similar, combinando información de fuentes académicas y comerciales para demostrar la caída persistente en el largo plazo de las reservas de capital social en Estados Unidos. Putnam da validez a la información recogida de varias fuentes frente a los hallazgos de la Encuesta Social General, reconocida ampliamente como una de las más fiables sobre la vida social americana (Ídem, Banco Mundial).

Es a partir de estos autores que surgen distintas vertientes del capital social, con distintas posturas teóricas, distintas temáticas empíricas. El alto reconocimiento de este paradigma se ve reflejado en los múltiples trabajos que se han publicado, con la finalidad de explicar una parte de la realidad cotidiana. Los organismos internacionales no son la excepción, por ello a continuación describiremos como éstos se involucran en el impulso de este campo de investigación, aunque su participación destaca en mayor medida en el desarrollo de trabajos empíricos.

\section{Aportes del campo para consolidar las relaciones sociales}

La aportación del campo del capital social se funda en la explicación de la relaciones sociales, con base en el marco de la acción colectiva (Ostrom, Ahn, \& Olivares, 2003). Este sirve de cimiento para las normas, las formas en la que se construyen las redes, las organizaciones y las instituciones que permiten la interacción social y el desarrollo de las sociedades, las cuales además generan una serie de efectos colaterales como las identidades o la información social. Reúne así, recursos benéficos para los individuos y las comunidades al existir lazos sociales que se traducirán en recursos financieros, también empodera al individuo frente la comunidad conforme aumentan sus lazos sociales, de solidaridad y reciprocidad (Bourdieu, 1986). Forma elementos socioculturales que son universales: memoria social, identidad, religión, vecindad, amistad, parentesco, reciprocidad, y elementos emocionales; estos factores contribuyen a la formación de capital social ${ }^{5}$ (Durston, 2003).

El capital social se convierte en un "recurso social" propio de los individuos ${ }^{6}$, que se alimenta por relaciones, redes e interacción social, y no se puede reproducir sino se pertenece a distintos grupos sociales. La intensidad de este tipo de interacciones es la que proporciona un alto capital 
social, las personas que sean ubicadas aquí tienden a desarrollarse en distintos ámbitos sociales que pueden ir desde la escuela, el trabajo, clubes, entre otros. En tanto las personas con bajo capital social ${ }^{7}$ sobrecargarán las pocas relaciones sociales que tengan, y exigirán mayores servicios públicos del Estado (UEOP, 2006; Flores \& Rello, 2003).

El capital social es un activo acumulable, el respeto de ciertas normas, brindar un grado de confianza a las demás personas, solidarizarse cuando alguien de la estructura social lo requiere, ser recíproco en la medida de lo posible con los apoyos recibidos, resultado de las relaciones sociales. Estas variables fortalecen a los individuos frente a los problemas sociales, fomentando las capacidades individuales y colectivas. Este escenario genera un ambiente propio para que la solidaridad, la cooperación, el asociacionismo, redes sociales se generen y permitan compartir información valiosa y disminuir así la incertidumbre acerca de las conductas de los otros. Esto a su vez se traduce en toma de decisiones colectivas sólidas, en donde los resultados de esas decisiones serán legitimas, y así se lograrán resultados equitativos para todos los participantes (Lechner, 2000; Lechner, 2002).Con la siguiente cita podemos resumir los posibles efectos positivos que puede tener el uso del capital social como forma de aproximarnos a la realidad.

“A medida que aumenta el capital social colectivo de un país, sus instituciones gozan de una mayor aceptación general y mejora la capacidad de negociación e intercambio. Por el contrario, en los países que tienen redes limitadas y desconectadas de capital social, las instituciones formales que suelen existir son reemplazadas por instituciones informales que dependen de relaciones personalizadas, y cuyas posibilidades de intercambio son más reducidas” (Robison, Siles, \& Schmid, 2003, pág. 53).

El capital social visibiliza una serie de problemas que las políticas públicas y las teorías dominantes no consideran, sobre todo porque al ser bienes no tangibles a diferencias de las dimensiones económicas, éstas últimas parecieran más importantes. Sin embargo, si se considera el funcionamiento de las relaciones sociales en los distintos grupos en el proceso de implementación de una política pública, se conocerán las reacciones y comportamientos de los grupos ante determinada política. Además que la parte social constituye una dimensión poco explorada de la calidad de vida que no tiene nada que ver con la estandarización de indicadores económicos (Bebbington, 2005).

\section{La perspectiva mundial del capital social}

\section{Instituciones internacionales y temáticas de asociación}

\section{Instituciones}

El concepto de capital social ha sido reconocido y retomado por algunas instituciones internacionales el Programa de Naciones Unidas para 
el Desarrollo (PNUD), la Comisión Económica para América Latina y el Caribe (CEPAL) y el Banco Interamericano del Desarrollo (BID) como el Banco Mundial (BM).

El PNUD ha impulsado reflexiones sobre el desarrollo que recuperan la perspectiva sociológica de las relaciones sociales, no sólo como medio de cohesión social, sino también como partícipes directos en los procesos productivos. Se pone énfasis en las relaciones entre personas, a diferencia de otras formas de capital que residen en los objetos (capital físico) o en los individuos de forma aislada (capital humano) (PNUD, 2008). Combinando la visión del desarrollo con el de las relaciones sociales este organismo presenta la siguiente visión:

"El capital social hace referencia a relaciones interpersonales que pueden ser utilizadas en la producción de bienes y servicios. De ellas es particularmente interesante su potencial como factor productivo, el grado en el que se utiliza y su rendimiento. Sin embargo, también lo es su contribución a las libertades de las personas, ya sea como medio para ampliar las oportunidades para elegir o al poner en juego la acción de los individuos como agentes de su propio desarrollo” (PNUD, 2008, pág. 97).

Este planteamiento vincula el capital social con el desarrollo humano al involucrar al individuo en la definición de sus objetivos, -teniendo la posibilidad de elegir entre varias opciones- que suponen necesariamente a otros, más aún cuando se trata de bienes públicos. Las oportunidades se amplían cuando hay relación con otros, es decir, el capital social altera la producción y el intercambio de bienes y servicios, modifica la disponibilidad de insumos para la generación de capacidades. De ello surge la necesidad de que se fomente la confianza, la cooperación y la institucionalización de las reglas como medio para alcanzar mayores niveles de desarrollo. En suma, se habla de capital social cuando los vínculos conforman una red relativamente sólida y activa de confianza y cooperación (PNUD, 2001; PNUD, 2008).

La concepción de la CEPAL pone acento en la inclusión de los individuos en posición de desigualdad y en la gestación de instituciones formales de asociación y participación. El trabajo sustancial se ha centrado en la inversión en el capital social ${ }^{8}$, que supone un efecto positivo en la reducción de la pobreza, la desigualdad y demás problemas sociales. De igual manera, la reducción de la pobreza y la desigualdad influye de manera positiva en el incremento de la capital social, porque se incentiva la cohesión social. La CEPAL (2002) propone que se considere el enfoque de capital social en el momento de promover las políticas públicas con la finalidad de romper con transmisión intergeneracional de oportunidades desiguales de bienestar. La pobreza aparece como un obstáculo a vencer en la región y es el capital social uno de los instrumentos que puede fortalecer el sector afectado. Aunado de un sistema económico dinámico e incluyente, además de un amplio sistema sociopolítico que sea consistente con los objetivos de 
inclusión (Atria, Siles, \& (Comps.), 2003; Arriagada \& (Ed.), 2005).

Así mismo, las reflexiones en el BID se dan, fundamentalmente, en dos sentidos: las contribuciones conceptuales sobre los vínculos entre capital social, valores éticos y cultura; y el fortalecimiento de la participación ${ }^{9}$ (Yamada, 2001). En la primera destaca Kliksberg (2000a y 2000b) que incluye el clima de confianza, el grado de asociatividad, la conciencia cívica, los valores éticos y, la cultura, entendida esta última como la "manera de vivir juntos" (Kliksberg B. , 2000a; 2000b).En la segunda, el BID destaca las formas de organización social de la región como las instituciones comunitarias, las autoridades tradicionales, las redes de parentesco y vecinos, organizaciones religiosas de servicio, y, otras, asociaciones voluntarias y de autoayuda que, por generaciones, han sido usadas por la población para movilizar recursos y atender fines de orden social, económico y político.

Todos estos medios organizativos son parte de la riqueza de los pueblos del continente y representan un importante acervo de capital social para la construcción de economías competitivas, sistemas políticos democráticos y sociedades más solidarias, sin pobreza ni exclusión (BID, 2000). En 2001 el BID presentó la Iniciativa Interamericana de Capital Social, Ética y Desarrollo, preparada bajo auspicios del gobierno noruego. Este trabajo impulsó el fortalecimiento de los valores éticos, y el capital social en los países de la región. Sin embargo, en la actualidad, al menos como aparece en el portal del BID, el proyecto se interrumpe.

Por su parte, el Banco Mundial ${ }^{10}$ también concibe al capital social ligado a las "normas de confianza", "los valores", "las actitudes y redes" entre personas e instituciones en un marco de "asociatividad" o de "acciones colectivas y de cooperación”. En lo individual, se refiere al grado de integración con su entorno a partir de las relaciones más cercanas de una persona, familia o empresa. A nivel sectorial, la acción se visualiza a través de personas u organizaciones en un entorno ampliado con los poderes públicos: pertenencia a redes comunitarias, gremios, asociaciones empresariales, etc. Finalmente, en la dimensión colectiva o nacional, el capital social se entiende como un acervo de una sociedad global. En ese sentido, el concepto de Capital Social agrupa nociones de confianza, asociatividad, conciencia cívica y valores éticos predominantes que, en conjunto, facilitan o dificultan el desarrollo de una determinada sociedad (Banco Mundial, 1999).

Como plantea el Banco Mundial, el "capital social” se liga temáticamente a las "normas de confianza", "los valores", "las actitudes y redes" existentes entre personas e instituciones, en el marco de la "asociatividad" o de las "acciones colectivas y de cooperación”. En lo individual, se refiere al grado de integración con su entorno a partir de las relaciones más cercanas de una persona, familia o empresa. A nivel sectorial, la acción se visualiza a través de personas u organizaciones en un entorno ampliado con los poderes públicos: pertenencia a redes comunitarias, gremios, asocia- 
ciones empresariales, etc...Finalmente, en la dimensión colectiva o nacional, el capital social se entiende como un acervo de una sociedad global. En ese sentido, el concepto de Capital Social agrupa nociones de confianza, asociatividad, conciencia cívica y valores éticos predominantes que, en conjunto, facilitan o dificultan el desarrollo de una determinada sociedad (Banco Mundial, 1998a; Banco Mundial, 1998b; Banco Mundial, 1999).

\section{Educación, salud y mercado ${ }^{11}$}

La educación recibida en la escuela es el segundo nivel de socialización — solo después que la familia - para los niños. El sentimiento de pertenencia, la participación, la corresponsabilidad, el trabajo en equipo o el acercamiento a la comunidad local nos hablan de calidad en los procesos, de la construcción del nosotros. Las escuelas son más efectivas cuando los padres y los ciudadanos locales se involucran en sus actividades. Los maestros están más comprometidos, los estudiantes alcanzan mejores resultados en los exámenes y se usan mejor las instalaciones de las escuelas en aquellas comunidades en las cuales los padres y ciudadanos se interesan en el bienestar educativo de los niños (Coleman y Hoffer 1987; Braatz y Putnam, 1996; Francis et al 1998) (Cfr. Portal del Banco Mundial).

La salud no es una condición que sólo depende del nivel de tecnología de los hospitales, la promoción de la salud está vinculada desde el eje individual hacia la consecución de cambios en el nivel comunitario y sistema social, está adquiriendo más fuerza y materializándose en nuevos programas. Algunos estudios sobre la salud han concluido que los problemas sociales de cohesión se ven traducidos en efectos físicos, llegando a la bien establecida conclusión de que un mejor nivel de salud depende de la distribución más equitativa de los ingresos (Sapag \& Kawachi, 2007).

Los médicos y enfermeras se presentan a trabajar y a cumplir sus obligaciones con más asiduidad en aquellos lugares donde sus actos son apoyados y supervisados por grupos de ciudadanos (Dreze y Sen, 1995). Sin embargo, a pesar de sus ventajas, el capital social también jugó un papel cuando las élites locales descontentas se unieron para cerrar centros de salud en Uttar Pradesh, India (Dreze y Sen, 1995). Como resultado, la tasa de mortalidad infantil aumentó una dolorosa advertencia de que el capital social también puede ser perjudicial para el desarrollo.

De un modo similar, el caso de Rusia ilustra como la falta de comprensión hacia formas preexistentes de capital social puede dificultar la adopción de políticas de implementación. Los esfuerzos rusos para privatizar las industrias estatales en un medio social donde la aplicación de la ley es débil y donde persisten facciones étnicas y nepotismo, ha creado una situación caracterizada por una desigualdad creciente, crímenes violentos y violación de los derechos humanos (Holmes, 1997).

Asegurar el acceso a los mercados es un paso crucial en el sendero hacia el avance económico de los pobres. Una de las características 
definitorias de ser pobre es la falta de conexiones con la economía formal incluido el acceso a los recursos materiales e informáticos. El capital social de los pobres se deriva primordialmente de la familia y de los vecinos, y puede servir como una red de seguridad cotidiana e importante, pero el capital social de los ricos les permite promover sus intereses. Ayudar a los pobres a trascender sus redes de seguridad a fin de que puedan tener acceso a recursos adicionales es uno de los desafíos del desarrollo económico.

\section{Asociaciones horizontales y verticales}

El capital social, en un sentido primario se le considera como "asociaciones horizontales" que incluyen redes sociales entre personas asociadas a normas que afectan a la productividad y el bienestar. Estas asociaciones pueden incrementar la productividad al reducir los costos asociados mediante el aprovechamiento de la coordinación y la cooperación aunque también pueden adoptar fines negativos: comunidades, grupos o redes que están aislados o tienen intereses exclusivamente locales o contrarios a los de una sociedad (por ejemplo, carteles de la droga, redes de corrupción) que pueden realmente frenar el desarrollo económico y social (Portes y Landholt 1996 Apud. TheWorld Bank, loc. cit.). Un sentido más comprensivo el capital social considera tanto los aspectos positivos como los negativos e incluye las asociaciones verticales y horizontales entre personas, incluyendo el comportamiento entre y dentro de las organizaciones, como por ejemplo las empresas. "Las relaciones horizontales son necesarias para dar un sentido de identidad y un propósito común a las comunidades, pero también (...) sin formar relaciones que transciendan varias divisiones sociales (por ejemplo, religión, etnia , estatus socio-económico) las relaciones horizontales pueden convertirse en una base para la búsqueda de intereses restringidos, que impidan el acceso a la información y a los recursos materiales que de otra manera pudieran ser de gran asistencia para la comunidad” (The World Bank, loc. cit.).

\section{Metodología: sociedad y política}

\section{Capacitación y Métodos}

Knack y Keefer (1997) usan indicadores de confianza y normas cívicas empleados en la Encuesta Mundial de Valores, que incluye una muestra de 29 economías de mercado. Se utilizan estas medidas como indicadores de la fortaleza de las asociaciones cívicas a fin de probar dos propuestas distintas sobre los efectos del capital social en el crecimiento económico: los "efectos Olson" (asociaciones de crecimiento restringido) y los "efectos Putnam" (asociaciones que facilitan el crecimiento a través de un incremento de la confianza). Ingelhart (1997) ha realizado el trabajo más extenso sobre las implicaciones de los resultados de la Encuesta Mundial de Valores para las teorías generales de la modernización y el desarrollo.

Narayan y Pritchett (1997) elaboran una medida del capital social 
en el sector rural de Tanzania utilizando cifras de la Encuesta sobre Capital Social y Pobreza de Tanzania (SCPS - Social Capital and Poverty Survey). Esta encuesta a gran escala preguntó a los individuos sobre el ámbito y las características de sus actividades asociativas y sobre su confianza en varias instituciones e individuos. Se compararon estas medidas de capital social con las cifras de ingresos familiares en pueblos similares (ambas del SCPS y de una encuesta previa sobre ingresos familiares, la Encuesta sobre el Desarrollo de Recursos Humanos). Se encontró que un mayor nivel de capital social en los pueblos incrementa los ingresos familiares.

Temple y Johnson (1998) ampliaron los trabajos previos de Adelman y Morris (1967) usando la diversidad étnica, la movilidad social y la extensión de los servicios telefónicos en varios países del África sub-sahariana como ejemplos de densidad de las redes sociales. Combinaron algunos elementos relacionados con un índice de "capacidad social” y mostraron que esto puede explicar una cantidad significativa de variantes en las tasas de crecimiento económico nacional.

Portes y Sensenbrenner (1993) examinan lo que sucede en las comunidades de inmigrantes cuando uno de sus miembros tiene éxito económico y desea abandonar la comunidad. Sus entrevistas revelan las presiones que los fuertes lazos comunitarios pueden ejercer sobre sus miembros; tan fuertes son esos lazos que algunos de sus miembros han cambiado sus nombres al inglés para liberarse de las obligaciones asociadas a su pertenencia a la comunidad. Gold (1995) provee información sobre cómo las comunidades judías en Los Ángeles tratan de mantener la integridad de la estructura comunitaria al tiempo que participan activamente en la vida económica.

Fernández-Kelley (1996) entrevista a muchachas en las comunidades de barrios marginales en Baltimore y descubre lo fuerte que son las presiones normativas para abandonar la escuela, tener un hijo en la adolescencia y rechazar un empleo formal. Rodeadas diariamente por la violencia, el desempleo, la adicción a droga, el único modo de establecer la identidad y el estatus de las jóvenes adolescentes es a través de sus cuerpos. Anderson (1995) estudió el papel de "las cabezas viejas”, los ancianos miembros de las comunidades pobres urbanas de la comunidad Afro-americana, como fuentes de capital social. Los “cabezas viejas” en cierta medida proveen sabiduría y orientación a los jóvenes, pero hoy en día sus consejos son cada vez más ignorados a causa del declive en el respeto a los ancianos y a que la comunidad continúa fragmentándose económicamente.

Heller (1996) estudió el caso del estado sureño de Kerala (India), donde las tasas de analfabetismo, longevidad y mortalidad infantil han sido las más favorables del sub-continente. Al examinar la historia de las relaciones estado-sociedad en Kerala, Heller demuestra como el estado ha tenido un papel crucial en la consecución de estos resultados por medio de la creación de condiciones que permiten a los grupos sociales subordinados organizarse en torno a sus intereses colectivos. Sin embargo, el estado de 
Kerala ha sido hostil a la inversión extranjera y al mantenimiento de la infraestructura, haciendo difícil para una población saludable y bien educada traducir su capital humano en una mayor prosperidad económica.

Portes (1995) y Light y Karageorgis (1994) examinan el bienestar económico de distintas comunidades de inmigrantes de los Estados Unidos. Demuestran que ciertos grupos (coreanos en Los Ángeles; chinos en San Francisco) prosperan mejor que otros (mexicanos en San Diego; dominicanos en Nueva York) debido a la estructura social a la que los nuevos inmigrantes se integran. Las comunidades exitosas son capaces de ofrecer ayuda a los recién llegados por medio de fuentes informales de crédito, seguro, apoyo a la niñez, capacitación en el idioma inglés y referencias de trabajo. Las comunidades menos exitosas muestran un compromiso a corto plazo con el país que los acoge y son menos capaces de proveer a sus miembros de servicios importantes.

Massey y Espinosa (1970) examinan la inmigración mexicana a los Estados Unidos. Señalan que políticas como NAFTA, que abogan por la libre circulación de bienes y servicios a través de las fronteras nacionales, también incrementan el flujo de personas, dado que los bienes y servicios son producidos, distribuidos y consumidos por el pueblo. Utilizando encuestas y datos recogidos a través de entrevistas, demuestran que una teoría del capital social es mejor predicción de hacia dónde emigrarán las personas, en qué número y por cuáles razones, que las teorías neoclásicas y de capital humano. Estos resultados son además usados como la base para proponer un número de medidas políticas innovadoras, destinadas a producir un manejo más equitativo y efectivo de la inmigración mexicana a los Estados Unidos.

\section{Proyectos de Desarrollo: diseño, ejecución e infraestructura}

Según el Banco Mundial el concepto de capital sociales útil en la implementación de proyectos de desarrollo tanto desde un punto de vista práctico como desde la experiencia en el terreno. También, es relevante en el debate teórico como en la práctica, especialmente, en el diseño e implementación de las políticas de desarrollo. En ellas el capital social juega un papel significativo, por ejemplo, cuando se habla de mejorar la educación, la salud, la la institucionalidad política y crear mercados abiertos en la medida que su utilización incide en la difusión y ampliación de la cooperación y los beneficios. Pese a que existen dificultades en su medición, el capital social se ha intentado traducir en indicadores cuantitativos. Existen definiciones amplias del capital social multidimensionales que incorporan distintos niveles y unidades de análisis a pesar de la ambigüedad de los conceptos como comunidad, red u organización que son sus referentes más inmediatos. Sin embargo, se han elaborado numerosos índices en encuestas, que intentan cuantificar la confianza en el gobierno, la intención de voto mediante, por ejemplo, la participación en organizaciones cívicas y el número de horas de voluntariado. Existe la esperanza que se ajusten los indicadores a través de nuevas técnicas y metodologías diversas: cualitati- 
vas, comparativas y cuantitativas. (Cfr. The World Bank, loc. cit.).

\section{Proyectos de desarrollo y capital social}

El conocimiento tácito es un elemento esencial en la elaboración de las políticas públicas como lo plantea tempranamente Dror. Una de sus formas más relevantes aparece en los sectores más modestos de la población, “acumulada”, como capital social. Este tipo de conocimiento resulta decisivo en el diseño y ejecución de los proyectos de desarrollo y asegura el énfasis hacia los sectores más vulnerables: crea más confianza y lealtad hacia el proyecto como lo demuestra en su trabajo sobre el desarrollo participativo en Sri Lanka Uphoffy de Tender en Brasil 1997(Cf. Narayan, 1995; Uphoff 1992; Tender 1997, Apud. The World Bank, loc. cit.).Los programas de microcréditos colectivos como el Banco Grame en Bangladesh son un ejemplo bastante conocido. Pero también los proyectos en otros sectores con vínculos intersectoriales obtienen resultados positivos: recursos financieros y políticos, administración mejor capacitada y acceso a apoyo técnico que redunda, en la eficacia y permanencia del proyecto. Pero, el capital social que existe entre varios actores se incrementa cuando se trascienden las divisiones de clase, género, etnia y religión: iniciativas en infraestructura como mejoras en los senderos para bicicletas o servicios de comunicaciones a bajo costo por ejemplo aumentan la capacidad de irradiación del capital social. A su vez, los micro proyectos dirigidos directamente a los pobres sólo pueden funcionar si se atienden los macro proyectos como los tendientes a mejorar las instituciones, el funcionamiento del sector público y las comunicaciones y evidentemente los dirigidos a mejorar el sistema judicial (ídem, The World Bank).

\section{Los desafíos del campo en las ciencias sociales}

Entre los distintos investigadores que se han aproximado a la realidad, mediante el paradigma del capital social, señalan la falta de acuerdo en la definición, medición y dimensiones. Sin embargo, a nuestro parecer el acuerdo de lo que significa el capital social parte con el reconocimiento de las relaciones sociales, como principal motor -como se presenta en el primer apartado-, seguida del establecimiento de dimensiones e indicadores que muestran su factibilidad, tales como la confianza y reciprocidad, solidaridad, generosidad, cooperación, etc. En el fondo hacen referencia a un mismo fenómeno, sin embargo, los distintos autores en algunas variables ponen un énfasis mayor, lo que no significa que descalifiquen las otras automáticamente.

\section{Dimensiones}

El principio para la generación y reproducción del capital social y de un conjunto de dimensiones e indicadores del capital social es la permanencia de los grupos y no de los individuos, aun cuando ellos sean los beneficiados. De ahí la diferencia con otros tipos de capital, en donde la 
acción individual es determinante para su acumulación. Los distintos planteamientos teóricos y las experiencias, -más allá de las ambigüedades que tiene el enfoque del capital social- indican que es un concepto multidimensional.

El principal componente del capital social es su composición, a diferencia de otros tipos de capital (humano, cultural, físico, económico) éste se desprende de las relaciones que se dan entre las personas. Esto significa una gran oportunidad, dado que no es posible encontrar individuos que estén totalmente aislados de las relacione sociales (López \& De la Torre, 2004). Esto permite que al relacionarse con otras personas haya posibilidades de reducir los costos de transacción, producir bienes públicos, y facilitar la constitución de organizaciones de gestión de base efectivas, de actores sociales y de sociedades civiles saludables (Durston, 2000).

El enfoque del capital social nos indica que pueden utilizarse este tipo de relaciones para coadyuvar al desarrollo de las capacidades de los individuos para superar los rezagos que presentan sobretodo en cuestiones de desigualdad y pobreza (Arriagada, 2003) y (Flores \& Rello, 2003). En este sentido, la relación que tiene con otros tipos de capital no es excluyente; por el contrario, es complementario, ya que al empoderarse en los distintos tipos de capital genera recursos para el individuo.

Estos recursos son potenciales y existen en todos los grupos humanos que están precedidos por elementos socioculturales aceptados en el imaginario social del grupo. Estos pueden ser la memoria social, identidad, religión, vecindad, amistad, parentesco, reciprocidad, y elementos emocionales. Dichos factores constituyen el bagaje cotidiano para la formación de capital social. Estos componentes ubican al capital social en un plano el plano conductual de las relaciones y sistemas sociales, y no en el plano abstracto de la cultura simbólica de las normas, los valores y las cosmovisiones, plano en el cual están el capital cultural y el capital humano o cognitivo. Ambos planos interactúan: ninguno de los dos determina siempre al otro (Durston, 2002).

El capital social es una capacidad que se desarrolla con las relaciones sociales. Sus beneficios inmediatos son la posibilidad de obtener beneficios a partir del aprovechamiento de redes sociales. La existencia de estas redes le brinda ventajas adicionales a los individuos que tienen acceso a ellas, en comparación con las que obtendrían si actuaran individualmente y sin el apoyo de esas relaciones sociales” (Flores \& Rello, 2003). Así pues llegamos a la formulación de dos definiciones sin pretender que son universales, ni mucho menos las más aceptadas, pero si nos dan un panorama de su concepción.

\section{Los principales indicadores}

Múltiples estudios se han realizado en los últimos años sobre el capital social, algunos generales, otros de casos específicos. Cada uno de 
ellos con el deseo de explicar lo que ocurre en la vida cotidiana. Creando sus propias herramientas para formular los indicadores que permitan aprehender la realidad, desmenuzarla y explicar cada uno de sus componentes. Sin embargo, al igual que en el aspecto teórico, del lado empírico no hay un consenso a la hora de definir cuáles son realmente los indicadores que reflejen los niveles de capital social. Sin embargo, debe reconocerse es el esfuerzo que se hace por llevar a cabo distintas investigaciones que permiten evaluar cualitativamente relaciones socioculturales específicas en los sectores y grupos sociales en condición de pobreza, haciendo posible mejorar el diseño de programas y proyectos, potenciando la confianza y la cooperación existentes y evitando conflictos entre los diversos agentes involucrados (Woolcock \& Narayan, 2001; Ocampo J. A., 2003).

Uno de los desafíos mayores para las ciencias sociales es generar herramientas que permitan aprehender la realidad, sea con técnicas cualitativas o cuantitativas. El enfoque del capital social tiene ese desafío, para general los indicadores viables operacionalmente, distinguiendo los distintos niveles para su análisis empírico como: a) las relaciones informales de confianza y cooperación; b) la asociación formal; y c) el marco institucional normativo y valorativo. También deben estar presentes las distintas formas positivas o negativas del capital social y al mismo tiempo distanciar al "bueno" del "malo" (Lechner, 2000; Stein, 2003). El poder establecer los indicadores comunes retroalimentaría al concepto, permitiendo mayor comprensión, ubicar los sectores más rezagos para invertir en ellos e incentivar este tipo de relaciones sociales, permite promover el financiamiento y promoción a través de las comunidades (Gallo Rivera \& Garrido Yserte, 2009).

La medición del capital social se vuelve un desafío para los científicos sociales, primero porque a diferencia de otras disciplinas no es un bien tangible, y segundo porque es un concepto joven, donde no hay uniformidad en su concepción y abordaje (Lechner, 2000; Lechner, 2002). Desde nuestro punto de vista el primer desafío será una constante, y sólo podrán mejorarse las técnicas de aproximación a la realidad y existirán unas más viables que otras y todo dependerá de los instrumentos que se utilicen para su estudio. Respecto al segundo punto, con los estudios realizados hasta la actualidad ya no podemos sostener dicha afirmación, dado que las discusiones teóricas con base en resultados empíricos se han disparado, esto ha hecho madurar el campo de investigación.

Así pues, pueden existir tantas listas de indicadores como investigadores dedicados a estos estudios, será recurrente en consecuencia preguntarnos por ejemplo si todos los individuos poseen capital social, qué indicadores son los más adecuados para aproximarnos a la realidad, cómo podemos distinguir el capital social "bueno" del "malo", con qué criterios se clasifican los distintos tipos de capital social, entre otras (Lechner, 2000; Stein, 2003). La realización de estudios empíricos ha hecho madurar el sentido teórico que se le ha asignado, metodológicamente se han tenido experiencias que enriquecen el paradigma, en términos prácticos se ha llamado la atención a los gobierno para que inviertan en las comunidades para 
fomentar este tipo de recursos (Gallo Rivera \& Garrido Yserte, 2009).

En las investigaciones que se han realizado, no se ha logrado establecer indicadores comunes. Se ha planteado más bien que es un concepto multidimensional, dado que incorpora diferentes niveles, unidades de análisis y los campos que se ven influenciados son diversos. Además varían de acuerdo a cada formulación teórica y experiencia que se tiene, debido a la evolución de las relaciones sociales y su vínculo con las organizaciones informales e instituciones formales. No se han dirigido esfuerzos en los estudios para medir el capital social de una forma común, por el contrario se han anclado a indicadores utilizados en otros índices y mediciones que van desde medidas de confianza, tendencias políticas, movilidad social (Arriagada, 2003; Flores \& Rello, 2003).

Respecto a la medición del capital social, en América Latina los estudios se han basado en la Encuesta Mundial de Valores, el Latinobarometro. El PNUD ha realizado varios esfuerzos para su medición como: Encuesta Nacional sobre Capital Social en el Medio Urbano (México 2006), Índice de Desarrollo Sociedad Civil, (Argentina, 1998), Encuesta Nacional PNUD (Chile, 1999). Cabe mencionar que en distintos países se han desarrollado encuestas nacionales y locales que miden algunas de las variables que se operacionalizan en el capital social, pero que no se mencionan explícitamente ${ }^{12}$.

Los resultados empíricos dejan pendiente una pregunta crucial: ¿Cómo producir capital social? Tan pronto nos interrogamos sobre cómo potenciar el capital social y fortalecer a la sociedad, desembocamos en un argumento circular: más capital social produce más sociedad y, de manera recíproca, una sociedad fuerte crea más capital social. Por lo tanto, no es el capital social el remedio milagroso que resucitara a la sociedad activa, cabe más bien suponer lo contrario: donde es débil la sociedad, también es débil la construcción de capital social (PNUD, 2001).

\section{Los desafíos del campo}

A pesar de su rápido éxito, el concepto de capital social presenta una serie de críticas, en distintos niveles. Una de ellas proviene de la teoría económica criticando algunos elementos básicos para llamar a algo "capital”. Keneth Arrow (2000) señala que la esencia de las redes sociales es que se construyen por razones distintas al valor económico que les adjudican sus participantes. Incluso esto es lo que les da su valor en el monitoreo. Ciertamente no encuentro consenso [...] para agregar algo llamado 'capital social' a las otras formas de capital (Arrow, 2000). También Solow (2000) ha dicho que no tiene mucho sentido hablar de capital, pues ideas como tasa de retorno o depreciación pierden significado en el contexto de las relaciones sociales: Si digo que la tasa de retorno del capital social ha caído del 10\% anual al 6\% anual desde 1975, ¿̇le dará esto algún panorama? [...] Dudo que el 'capital social' sea el concepto correcto para discutir lo que sea que estamos discutiendo (Solow, 2000). 
Los planteamientos no son novedosos porque refiere a la sociedad tal como funciona, reivindicando su carácter moral y legitimando, con ello, un orden social particularista y desigual. Aparejado a lo anterior, hay un retroceso en el funcionamiento del Estado y del mercado al legitimar los lazos familiares, comunales y locales, como fundamento del reconocimiento y la distribución de la riqueza social. Ha reconocido algunas causas de desigualdad y promete solucionarlo con lazos sociales que se restringen a los grupos pequeños de la familia, los amigos o el vecindario, sin enfrentarse a los problemas estructurales como los privilegios a las élites (Marrero, 2006).

La ambigüedad de los indicadores para medirla, se ha hecho evidente, ello sobre todo porque es un capital o un bien no tangible y un concepto que se está desarrollando y aprendiendo de la experiencia. Por esta razón, la carencia de un marco teórico desemboca en problemas metodológicos para cuantificar empíricamente el fenómeno. Resulta difícil ponderar la significación de los datos, pues no son comparables con cifras de otros países ni existen series temporales (Lechner, 2000; Lechner, 2002). Los resultados empíricos dejan pendiente una pregunta crucial: ¿Cómo producir capital social? Tan pronto nos interrogamos sobre cómo potenciar el capital social y fortalecer a la sociedad, desembocamos en un argumento circular: más capital social produce más sociedad y, de manera reciproca, una sociedad fuerte crea más capital social. Por lo tanto, no es el capital social el remedio milagroso que resucitara a la sociedad activa, cabe más bien suponer lo contrario: donde es débil la sociedad, también es débil la construcción de capital social (PNUD, 2001; Robison, Siles, \& Schmid, 2003).

Los alcances del capital social, pueden también conducir a la discriminación y exclusión de las personas o grupos que no formen parte de él ${ }^{13}$. Además las distintas formas de capital social puede conducir a las personas o grupos a actuar en detrimento de las instituciones y normas sociales que generan gastos públicos en beneficio de los pocos miembros de sus redes ricas en capital social (Robison, Siles, \& Schmid, 2003; Fukuyama, 2003). Excesivas exigencias a los miembros del grupo y restricciones a la libertad individual; el caso de redes demasiado cerradas donde la obtención de beneficios comunes, crea restricciones para la iniciativa individual, y donde la responsabilidad individual es sustituida por la responsabilidad del grupo (Gallo Rivera \& Garrido Yserte, 2009).

\section{Conclusiones}

En las investigaciones sobre capital social, no se ha logrado establecer indicadores comunes. Se ha planteado más bien que es un concepto multidimensional, dado que incorpora diferentes niveles, unidades de análisis y los campos que se ven influenciados son diversos. Además varían de acuerdo a cada formulación teórica y experiencia que se tiene, debido a la evolución de las relaciones sociales y su vínculo con las organizaciones 
informales e instituciones formales. No se han dirigido esfuerzos en los estudios para medir el capital social de una forma común, por el contrario se han anclado a indicadores utilizados en otros índices y mediciones que van desde medidas de confianza, tendencias políticas, movilidad social.

Los resultados empíricos dejan pendiente una pregunta crucial: ¿Cómo producir capital social? Tan pronto nos interrogamos sobre cómo potenciar el capital social y fortalecer a la sociedad, desembocamos en un argumento circular: más capital social produce más sociedad y, de manera recíproca, una sociedad fuerte crea más capital social. Por lo tanto, no es el capital social el remedio milagroso que resucitara a la sociedad activa, cabe más bien suponer lo contrario: donde es débil la sociedad, también es débil la construcción de capital social. 


\section{Notas}

${ }^{1}$ Los orígenes han sido citados por múltiples autores desde los fundadores como Putman, pasando por Fukuyama y considerado en los en múltiples estudios.

${ }^{2}$ Desde la fundación de la sociología, Durkheim enfatizó la necesidad y el funcionamiento de las relaciones sociales tanto para la sociología como para la economía, aunque el fin que deseaba obtener en una era distinta a la otra, en esencia se daban de la misma manera, por ejemplo señalaba “...que el intercambio mercantil presupone la existencia de normas sociales no económicas que dictan, por ejemplo que los compradores y vendedores negociarán pacíficamente en lugar de sacar un arma y tratar de robarse y asesinarse mutuamente” (Apud. Fukuyama, 1999, pg. 199).

${ }^{3}$ Para una descripción general de la bibliografía escrita hasta 1999 consultar (Rossing Feldman \& Assaf, 1999).

${ }^{4}$ La tradición intelectual de la que proviene cada autor, explican los rasgos fuertes, distintivos: Coleman, quien inscribe su trabajo en la tradición de la elección racional, postula una teoría de la acción basada en el intercambio social. Putnam, por su parte, se adhiere a una perspectiva republicana, ligada a las tradiciones políticas que apelan al carácter virtuoso de las relaciones (Millán \& Gordon, 2004).

${ }^{5}$ Siguiendo a Woolcook (2001) existen al menos siete campos en los se ha venido utilizando la teoría del capital social: 1) la familia y los jóvenes; 2) la escuela y la educación; 3) la vida de las comunidades; 4) el trabajo y las organizaciones; 5) la democracia y la gobernanza; 6) los problemas de la acción colectiva; y 7) el desarrollo económico (Gallo Rivera \& Garrido Yserte, 2009).

${ }^{6}$ El capital social se desarrolla fundamentalmente en: la familia, primera instancia de socialización; las comunidades al interactuar por el bien común; las empresas al formar redes de confianza en la organización; la sociedad civil al ofrecer oportunidades de participación; el Estado al buscar el bienestar de la sociedad por mencionar algunas.

${ }^{7}$ Algunas reflexiones en las ciencias sociales se han dirigido a indagar sobre la cuantificación o medición de distintos indicadores. En lo que respecta al capital social la discusión sobre cuáles son los indicadores y las dimensiones adecuadas que permiten acercarse más a la realidad son múltiples y por ello, menos son los acuerdos de que variables se deben considerar. Sin embargo, en la práctica se ha utilizado la Encuesta Mundial de Valores, el Latinobarometro, y encuestas locales de cada país en las que se han utilizado variables como: confianza social, compromiso cívico, normas de reciprocidad, relaciones sociales, participación en organizaciones, acción colectiva, percepciones sobre el respeto a los derechos y a la ley, redes sociales, etc.

${ }^{8}$ Tres son los principales componentes del capital social: 1) las fuentes y la infraestructura, es decir, el rol de la cultura (valores, normas y costumbres internalizadas desde la infancia)

2) los determinantes que impone la estructura social (interacciones sociales que generan reglas del juego y normas institucionalizadas) y 3) las consecuencias y resultados de estas acciones, que pueden ser positivas o negativas (Flores \& Rello, 2003).

${ }^{9}$ Uno de los primeros aportes conceptuales del BID sobre el tema de capital social provino de la conferencia sobre el tema convocada en su Cuadragésima Asamblea Anual de Gober- 
nadores en 1999 en París. Las más importantes contribuciones a ella están compiladas en el libro "Capital social y cultura: claves estratégicas para el desarrollo" (Kliksberg y Tommasini, 2000) cuya contribución central es la reflexión sobre el papel de los aspectos valóricos del capital social en el desarrollo.

${ }^{10}$ En 1996 crea la "Social Capital Initiative” (SCI) adscrita al Departamento de Desarrollo Social del Banco Mundial, esta Iniciativa de Capital Social (SCI) incluye 12 proyectos de investigaciones originales, un marco conceptual, revisiones de literatura, bibliografías anotadas, y actividades asociadas. Sus objetivos son 1) evaluar el impacto del capital social; 2) demostrar que la ayuda exterior puede ayudar en el proceso de formación de capital social; y 3) contribuir al desarrollo de indicadores para el seguimiento del capital social y metodologías para la medición de su impacto en el desarrollo.

${ }^{11}$ Lo que sigue ha sido elaborado a través de la información del portal del Banco Mundial.

${ }^{12}$ No se hace un intento por reunir cada una de ellas, pero se presentan algunas como: la Encuesta Nacional de Cultura Política y Prácticas Ciudadanas (México); Encuesta Nacional sobre Capital Social en el Medio Urbano, (Chile 2000 y México 2006), Encuesta Nacional sobre Filantropía y Sociedad Civil (México). Además se suman esfuerzos de la Fundación BBVA (España), de la Encuesta Mundial de Valores y el Latinobarómetro.

${ }^{13}$ Durston (2000) señala que algunos efectos negativos para las personas y las colectividades, son: sus efectos restrictivos de la libertad, su carácter excluyente y la rivalidad entre líderes, grupos que se basan en la amenaza de violencia más que en la confianza. 


\section{Bibliografía}

Arriagada, I., \& (Ed.) (2005), Aprender de la experiencia. El capital social en la superación de la pobreza. Naciones Unidas, CEPAL y Cooperazione Italiana, Santiago de Chile.

Arrow, K. (2000),“Observations on social capital”. En P. Dasgupta, \& I. Serageldin, Social Capital: A Multifaceted Perspective. The World Bank, Washington.

Atria, R. (2003), “Capital social: concepto, dimensiones y estrategias para su desarrollo”. En R. Atria, \& M. e. Siles, Capital social y reducción de la pobreza en América Latina y el Caribe: en busca de un nuevo paradigma (págs. 581-590). CEPAL-Universidad del Estado de Michigan, Chile.

Atria, R.; Siles, M. \& et al. (2003), Capital social y reducción de la pobreza en América Latina y el Caribe: en busca de un nuevo paradigma. CEPALUniversidad del Estado de Michigan, Chile.

Banco Mundial (1999), Capital Social en Español. Recuperado el septiembre de 2010, de http://web.worldbank.org

Ídem (1998a), “The Initiative on Defining, Monitoring and Measuring Social Capital: Overview and Program Description”. Social Capital Initiative, Working Paper No. 1 (pág. 45). Banco Mundial, Washington D.C.

Ídem (1998b), The Initiative on Defining, Monitoring and Measuring Social Capital: Text of Proposals Approved for Funding. Social Capital Initiative, Working Paper No. 2 (pág. 163). Wasington D.C.: Banco Mundial.

Bebbington, A. (s/f), "El capital social en el desarrollo: teoría, concepto o estrategia?”. En CEPAL, SERIE Seminarios y conferencias (págs. 31-38). CEPAL, Chile.

Ídem (2005), “Estrategias de vida y estrategias de intervención: el capital social y los programas de superación de la pobreza”. En I. Arriagada, \& (Coord), Aprender de la experiencia. El capital social en la superación de la pobreza (págs. 21-46). CEPAL, Chile.

Becker, G. (1976), The Economic Approach To Human Behavior. University of Chicago Pre, Chicago.

BID. (2000), Marco Estratégico para la Participación Ciudadana en las Actividades del Banco Interamericano de Desarrollo. Obtenido de Disponible en http://www.iadb.org/etica/iniciativa.cfm

Boisier, S. (2003), Comunicación al seminario-taller "El largo brazo de Descartes: usos y abusos del concepto de capital social en las propuestas 
de desarrollo”. En I. Arriagada \& F. Miranda (Comps), Capital social: potencialidades analíticas y metodológicas para la superación de la pobreza (págs. 61-68). CEPAL, Chile.

Bourdieu, P. (1986), “The forms of capital”. En J. Richardson, Handbook of Theory and Research for the Sociology of Education. Greenwood Press, New York.

Claridge, T. (2004), Social capital reserch. Recuperado el 09 de Noviembre de 2010, de http://www.socialcapitalresearch.com

Coleman, J. (1990), Foundations of social theory. Belknap Press, Cambridge.

Ídem (1988), “Social capital in the creation of human capital”. The American Journal of Sociology Vol. 94, S95-S120.

Cuéllar Saavedra, Ó. \& Bolívar Espinoza, A. G. (2009), Capital social hoy. Polis .

Downs, A. (1957), An Economic Theory of Democracy. Harper, New York.

Durkheim, É. (2005), La división social del trabajo. Colofón, México.

Durston, J. (2000), ¿Qué es el capital social comunitario?, CEPAL, Chile.

Ídem (2003), “Capital social: parte del problema, parte de la solución, su papel en la persistencia y en la superación de la pobreza en América Latina y el Caribe”. En R. Atria \& M. e. Siles, Capital social y reducción de la pobreza en América Latina y el Caribe: en busca de un nuevo paradigma (págs. 147-202). CEPAL-Universidad del Estado de Michigan, Chile.

Ídem (2003), Capital social: parte del problema, parte de la solución, su papel en la persistencia y en la superación de la pobreza en América Latina y el Caribe. En R. Atria, \& e. al, Capital social y reducción de la pobreza en América Latina y el Caribe: en busca de un nuevo pardigma (págs. 147-202). CEPAL, Chile.

Ídem (2002), El capital social campesino en la gestión del desarrollo rural. Díadas, equipos, puentes y escaleras. CEPAL, Chile.

Flores, M., \& Rello, F. (2003), “Capital social: virtudes y limitaciones”. En R. Atria, \& e. a. (Comps), Capital social y reducción de la pobreza en América Latina y el Caribe: en busca de un nuevo paradigma (págs. 203227). CEPAL-Universidad del Estado de Michigan, Chile.

Fukuyama, F. (2003), “Capital social y desarrollo: la agenda venidera”. En R. Atria, \& M. e. Siles, Capital social y reducción de la pobreza en América Latina y el Caribe: en busca de un nuevo paradigma (págs. 33-50). 
CEPAL-Universidad del Estado de Michigan, Chile.

Fukuyama, F. (1999), La gran ruptura. Atlantida, Madrid.

Gallo Rivera, M. T. \& Garrido Yserte, R. (2009), El capital social. ¿Qué es y por qué importa? IUAES-Universidad de ALcalá, Madrid.

Kliksberg, B. (2000b), Capital social y cultura. Claves olvidadas del desarrollo. BID-INTAL, Argentina.

Ídem (2000a), "El rol del capital social y de la cultura en el proceso de desarrollo”. En B. Kliksberg \& L. Tomassini, Capital social y cultura: claves estratégicas para el desarrollo. BID, Washington.

Lechner, N. (2000), "Desafíos de un desarrollo humano: individualización y capital social”. En B. Kliksberg, \& L. Tomassini, Capital Social y Cultura: Claves estratégicas para el desarrollo (págs. 19-58).BID-FCE, Argentina.

Ídem (2002), “El capital social como problema cultural”. Revista Mexicana de Sociología, 91-109.

López, P., \& De la Torre, R. (2004), Capital social y desarrollo humano en México. PNUD, México.

Ocampo, J. A. (2003), “Capital social y agenda del desarrollo”. En R. Atria \& M. e. Siles, Capital social y reducción de la pobreza en América Latina y el Caribe:en busca de un nuevo paradigma (págs. 25-31). CEPAL-Universidad del Estado de Michigan, Chile.

Ídem (2005), Más allá del Consenso de Washington: una agenda de desarrollo para América Latina. CEPAL, México.

Olson, M. (1965), The logic of Collective Action. Public Goods and Theory of Groups. Harvard University Press, Cambridge.

Ostrom E.; Ahn, T. \& Olivares, C. (2003), "Una perspectiva del capital social desde las ciencias sociales: capital social y acción colectiva”. Revista Mexicana de Sociología, Vol. 65, No. 1, 155-233.

PNUD (2001), Desarrollo humano en Chile 2000. Más sociedad para gobernar el futuro. PNUD, Chile.

Ídem (2008), Informe sobre desarrollo humano. Michoacán 2007. PNUD, México.

Putman, R. D. (2003), El declive del Capital Social. Un estudio internacional sobre las sociedades y el sentido comunitario. Galaxia Gutenberg, Barcelona. 
Putnam, R. (1993), Making Democracy Work: Civic Traditions in Modern Italy. Princeton University Press, Princeton.

Robison, L. J.; Siles, M. E. \& Schmid, A. A. (2003), “El capital social y la reducción de la pobreza: hacia un paradigma maduro”. En R. Atria \& M. e. Siles, Capital social y reducción de la pobreza en América Latina y el Caribe: en busca de un nuevo paradigma (págs. 51-114). CEPAL-Universidad del Estado de Michigan, Chile.

Rossing Feldman, T. \& Assaf, S. (1999), "Social Capital: Conceptual Frameworks and Empirical Evidence; An Annotated Bibliography”. Social Capital Initiative, Working Paper No. 5 (pág. 49). Banco Mundial, Washinton D.C.

Sen, A. (1973), On Economic inequality. Charenton Pre, Oxford.

Sen, A. \& Foster, J. E. (1997). La desigualdad económica. Edición ampliada con una anexo fundamental de E. Foster y Amartya Sen. Alianza, Madrid.

Solow, R. (2000), "Notes on Social Capital and Economic Performance”. En P. Dasgupta, \& I. Serageldin, Social Capital: A Multifaceted Perspective. The World Bank, Washington

Stein, R. H. (2003), Capital social, desarrollo y políticas públicas en la realidad latinoamericana. Universidad de Brasilia, Brasil.

Tello, M. (2006), Aspectos teóricos del Capital Social y elementos para su uso en el análisis de la realidad. Consorcio de Investigaciones Económicas (CIES), Perú.

UEOP, U. d. (2006), Capital social: confianza, redes y asociacionismo en 13 países del mundo. Fundación BBVA, España.

Weber, M. (2000), Economía y sociedad. FCE, México.

Woolcock, M., \& Narayan, D. (2001), Capital social: Implicaciones para la teoría, la investigación y las políticas sobre desarrollo. Documento del Banco Mundial, Washington.

Yamada, G. (2001), Reducción de la pobreza y fortalecimiento del capital social y la participación: La acción reciente del Banco Interamericano de Desarrollo. Capital Social y Pobreza (pág. 22). CEPAL, Santiago de Chile. 